\title{
A Lemma on the Structure of Lie Algebras and the Problem of the Combination of Relativistic and Internal Symmetries.
}

\author{
V. BERzI \\ Istituto di Scienze Fisiche dell'Università - Milano \\ Istituto Nazionale di Fisica Nucleare - Sezione di Milano \\ (Lett. Nuovo Cimento, 2, 156 (1969))
}

On page 158:

fourth line instead of $1_{B}$ and $B$ please read $1_{A}$ and $A$.

fifth line: the formula should be read as:

$\pi \operatorname{ad} p \operatorname{ad} q=\pi \operatorname{ad} p\left(1_{\Lambda}-\pi+\pi\right)$ ad $q=\pi \operatorname{ad} p \pi \operatorname{ad} q+\pi \operatorname{ad} p\left(1_{\Lambda}-\pi\right)$ ad $q$. sixth line $p(r)$ should be replaced by $q(r)$ and $1_{B}$ by $1_{A}$. 